\title{
Comparison of basal-like triple-negative breast cancer defined by morphology, immunohistochemistry and transcriptional profiles
}

Patrycja Gazinska ${ }^{1,2,5}$, Anita Grigoriadis ${ }^{1,5}$, John P Brown ${ }^{2}$, Rosemary R Millis ${ }^{2}$, Anca Mera ${ }^{3,4}$, Cheryl E Gillett ${ }^{3}$, Lars H Holmberg ${ }^{3,4}$, Andrew N Tutt ${ }^{1}$ and Sarah E Pinder ${ }^{2}$

${ }^{1}$ Breakthrough Breast Cancer Research Unit, King's College London, School of Medicine, Division of Cancer Studies, Bermondsey Wing, Guy's Hospital, London, UK; ${ }^{2}$ Department of Research Oncology, King's College London, School of Medicine, Division of Cancer Studies, Bermondsey Wing, Guy's Hospital, London, UK;

${ }^{3}$ Breast Tissue and Data Bank, King's College London, School of Medicine, Division of Cancer Studies, Bermondsey Wing, Guy's Hospital, London, UK and ${ }^{4}$ Cancer Epidemiology Group, King's Health Partners AHSC, King's College London, School of Medicine, Division of Cancer Studies, Bermondsey Wing, Guy's Hospital, London, UK

Basal-like invasive breast cancer is an important clinical group because of its association with a triple-negative phenotype defined by the lack of expression of estrogen, progesterone and human epidermal growth factor receptors 2, relative lack of therapeutic options and poor prognosis. However, depending on the method used to define these lesions, morphological assessment, immunohistochemical markers or gene expression, a different set of tumors is captured. The aim of this study was to investigate the consequences of using different methodological approaches to define basal-like lesions among triple-negative breast carcinomas with regard to their clinicopathological features and patient outcome. The cohort consisted of 142 invasive breast cancers with a triple-negative receptor status. First, each was reviewed histologically and those with morphological basallike features were characterized as 'Path-Basal'. Second, the 'Core Basal' immunohistochemical lesions, defined as cytokeratin $5 / 6$ and/or epidermal growth factor receptor 1 positive, within the triple-negative breast cancers were identified, and third their classification based on gene expression profiling was retrieved and those in the molecular 'PAM50 basal-like' subtype recorded. A total of 116 basal-like breast cancers were identified among the 142 triple-negative breast cancers by at least one of these three classifications ( $80 \%)$, but only 13 samples were defined as basal-like with all three methods. None of these 13 tumors were associated with lymphovascular invasion. The 34 morphological 'Path-Basal' lesions were significantly associated with a lack of nodal metastases. Comparing the estimates of death in the three classifications, the highest risk of death was seen for the 'Core Basal' group. In this study, we highlight that the definition of basal-like breast cancer based on different methodologies varies significantly and does not identify the same lesions. This incomplete overlap of cases emphasizes the need for consistent or new approaches to improve precise identification.

Modern Pathology (2013) 26, 955-966; doi:10.1038/modpathol.2012.244; published online 8 February 2013

Keywords: basal-like phenotype; invasive breast cancer; triple-negative breast cancer

Correspondence: Professor SE Pinder, FRCPath, King’s College London, School of Medicine, Research Oncology, 3rd Floor Bermondsey Wing, Guy's Hospital, Great Maze Pond, London SE1 9RT, UK.

E-mail: sarah.pinder@kcl.ac.uk

${ }^{5}$ These authors contributed equally to this work.

Received 2 October 2012; revised 20 November 2012; accepted 22

November 2012; published online 8 February 2013
Breast cancer is a heterogeneous disease, which can be classified into biologically, morphologically and clinically meaningful entities. Approximately 12 to $17 \%$ are triple-negative breast cancers lacking expression of estrogen receptor (ER), progesterone receptor (PR) and human epidermal growth factor receptor 2 (HER2). ${ }^{1}$ These lesions are also characterized by a high incidence of TP53 mutations, as well as low levels of $\mathrm{RB}$ and high 
levels of p16 proteins. ${ }^{2,3}$ Triple-negative breast cancers are more prevalent in patients below the age of 50 years ${ }^{4}$ who are of African-American ethnicity ${ }^{5}$ and who have a more aggressive clinical behavior $^{6-8}$ as well as a distinctive metastatic pattern. ${ }^{9}$ In particular, triple-negative breast cancers are clinically problematic as there is no approved targeted systemic therapy for these lesions; in some series, patients with triplenegative breast cancers have an increased risk of recurrence between the first and third years after diagnosis and an increased mortality in the first 5 years after treatment. Currently, chemotherapy is the only systemic therapy available for triple-negative breast cancers and is curative in a subset of patients with chemotherapy-sensitive disease. ${ }^{10}$

Genomic studies such as the PAM50 gene expression assay have demonstrated that breast cancers can be classified into at least five intrinsic subtypes (luminal A, luminal B, HER2-enriched, basal-like and normal breast-like). ${ }^{11}$ Triple-negative breast cancers overlap with the molecular entity of 'basallike' breast cancers, ${ }^{12}$ but these are not the same entity and equating them is misleading. ${ }^{13-15}$ Many current studies make use of microarray-based expression profiling to define basal-like breast cancers; however, continuous efforts are being made to define these lesions with standard pathological techniques, for example, using immunohistochemical markers as surrogates. ${ }^{7,16,17}$ With the latter approach, it has been reported that $\sim 50 \%$ of triple-negative breast cancers are either positive for epidermal growth factor receptor 1 (EGFR) and/or basal cytokeratin (CK) 5/6 and have been referred to as 'Core Basal'. ${ }^{16}$ Among triple-negative breast cancers treated with adjuvant anthracycline-based chemotherapy, the 'Core Basal' phenotype has been reported to be associated with a significantly worse outcome. ${ }^{16}$

Classically, they are high-grade lesions of ductal/ no-special type, mostly having a high mitotic index, lack of tubule formation and marked cellular pleomorphism. ${ }^{14,18}$ The nuclear chromatin pattern ranges from coarse to vesicular, and prominent nucleoli are commonly present. ${ }^{19} \mathrm{~A}$ pushing, or partially pushing, margin and a peritumoral lymphocytic infiltrate are frequently described. ${ }^{18,20}$ In particular, large zones of geographic necrosis, frequent apoptotic cells, stromal lymphocytic infiltrates and scanty stromal content are characteristic. ${ }^{21}$ Occasionally, these tumors also contain areas of spindle cell and squamous metaplasia. ${ }^{14,22}$ From a morphologic point of view, basal-like breast carcinomas are clearly a heterogeneous group of cancers, including medullary, metaplastic and spindle cell, myoepithelial and adenoid cystic tumors. ${ }^{14}$

As there is presently no consensus regarding the optimal definition of basal-like triple-negative breast cancers, our aim was to explore the clinicopathological features of basal-like breast cancers when defined by molecular, immunohistochemical and morphological approaches. A cohort of consecutive triple-negative breast cancer patients with extensive clinical information was reviewed histopathologically and subjected to a panel of immunohistochemical biomarkers characterizing 'Core Basal' lesions. In addition, assignment to the molecular basal-like PAM50 subtype on the basis of their expression profiles was retrieved and comparison between these three classifications was made.

\section{Materials and methods}

\section{Patient Demographics}

This study encompassed a retrospective consecutive series of 142 female patients identified as having primary invasive triple-negative breast carcinoma diagnosed between 1971 and 2001 and treated at Guy's and St Thomas' Hospitals (London, UK), and none of whom had prior neoadjuvant therapy. Ethical approval for the analysis of tissue samples and patient data was obtained from the local research ethics committee and in accordance with the ethical principles expressed in the Declaration of Helsinki. Access to the pseudo-anonymized samples and clinical data was obtained in accordance with the principles of the Guy's and St Thomas' Research Tissue and Data Bank (REC No 07/H0804/131).

The median age of patients at the time of diagnosis was 55 (24-87 years). Axillary lymph node clearance was performed in 117 (82\%) patients, of whom $55(38 \%)$ had at least one positive lymph node. Of the 139 patients without distant metastasis at diagnosis, 127 received adjuvant therapy; this comprised adjuvant radiotherapy in $86(60 \%)$, adjuvant chemotherapy in $57(40 \%)$ and adjuvant endocrine therapy in $63(44 \%)$ patients. Sixty (47\%) patients had more than one treatment and 12 (9\%) patients received all three. Median follow-up, defined as the date of diagnosis to the last known follow-up or death, was 8.3 years (range 0.3-22.2, mean 8.9 years). All women were followed-up and the data were prospectively entered into the Guy's and St Thomas' Research Tissue and Data Bank. Forty-seven (33\%) patients developed distant metastasis; the median time to metastasis, from diagnosis of the primary tumor, was 1.6 years (range 0.2-18.7). Forty-eight patients died from breast cancer, with a median overall survival of 2.5 years (mean 3.7, range $0.3-19.2$ years).

\section{Histological Assessment}

Sections from the surgical excisions of 142 women with triple-negative breast cancers were retrieved from the Guy's and St Thomas' Breast Tissue and Data Bank, Guys' Hospital, (London, UK). Sections were reviewed by a specialist consultant breast pathologist (SEP). Specifically, invasive tumor size, histologic grade and subtype, presence of associated ductal carcinoma in situ (DCIS) and of 
lymphovascular invasion and nodal status were assessed. ${ }^{23}$ This was based on examination of all available hematoxylin and eosin-stained sections without knowledge of immunohistochemical or microarray results.

Tumors reported as invasive ductal/no-special type with basal-like features ${ }^{9,18}$ (Figure 1a1 and a2) included those with a solid growth pattern and a pushing margin of invasion with scanty intervening stroma. An associated lymphocytic-rich stroma (Figure 2c and d) and extensive geographic areas of necrosis were observed in the majority of cases (Figure 2b). Carcinoma cells were highly pleomorphic and proliferative, often with atypical mitoses, and tubule formation was absent. Apoptotic cells were typically numerous and close to areas of necrosis. A central fibrotic zone was frequent (Figure 2a). Medullary-like (Figure 1b1 and b2) and metaplastic tumors (Figure 1c1 and c2) were also included in the morphologic basal-like group.

\section{Tissue Microarrays and Immunohistochemistry}

Representative areas of the donor block were marked on a stained section. Tissue microarrays were constructed, using the Beecher microarrayer (Sun Prairie, WI, USA) with $0.6 \mathrm{~mm}$ stylets. Tissue microarrays were made in triplicate mainly from the periphery of the carcinoma and also other representative areas. Analysis and scoring of tissue microarrays was carried out by two observers (SEP and PG). Expression of ER, PR and HER2, CK5/6,
CK14 and EGFR was assessed. Antibodies are listed in Table 1 and the scoring system in the Table $2 .^{7,24}$ Using a system equivalent to the five biomarker method, ${ }^{16}$ triple-negative breast cancers were categorized into those positive for EGFR and/or CK5/6, referred to as the 'Core Basal', and those of a five marker negative phenotype $(5 \mathrm{NP}=$ negative for ER, PR, HER2, CK5/6 and EGFR).

\section{Molecular Subtyping Based on Gene Expression}

Gene expression profiles of the 142 triple-negative breast cancers were recorded as part of a larger study comprising 205 breast tumors, including other breast cancer intrinsic subtypes (the extended cohort is described in detail in Rinaldis et al, under review). To retrieve the classification of the molecular PAM50 subtypes, we performed an advanced nearest centroid classification using the class centroids from Parker et al. ${ }^{25}$ Briefly, we randomly sampled tumors from the larger 205 cohort, creating 10000 artificial cohorts with compositions of ERpositive, HER2-positive, ER- and HER2-negative and normal-like breast tumors ${ }^{25}$ (see Supplementary Methods). Assignment of tumors to the molecular subtypes was based on their highest Spearman's rank correlation.

\section{Statistical Analysis}

All statistical analyses were performed in the $\mathrm{R}$ environment, using several CRAN packages (http://
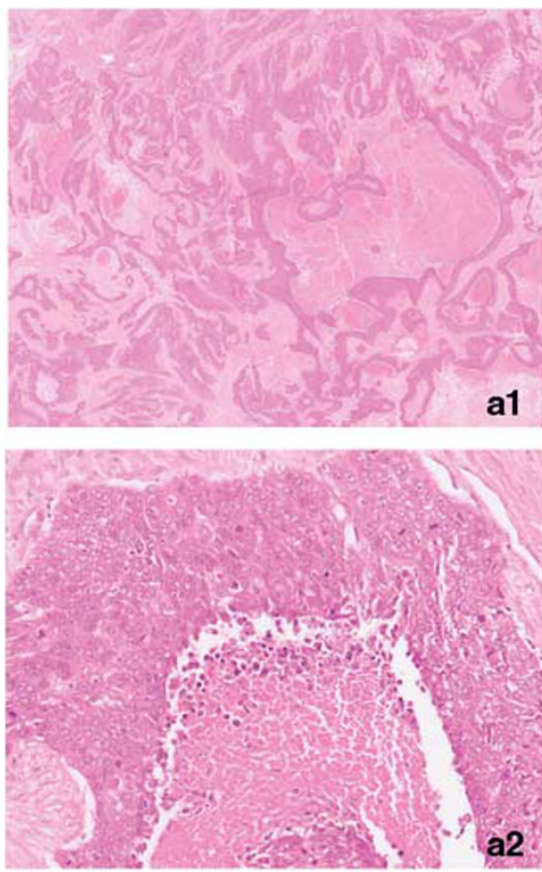
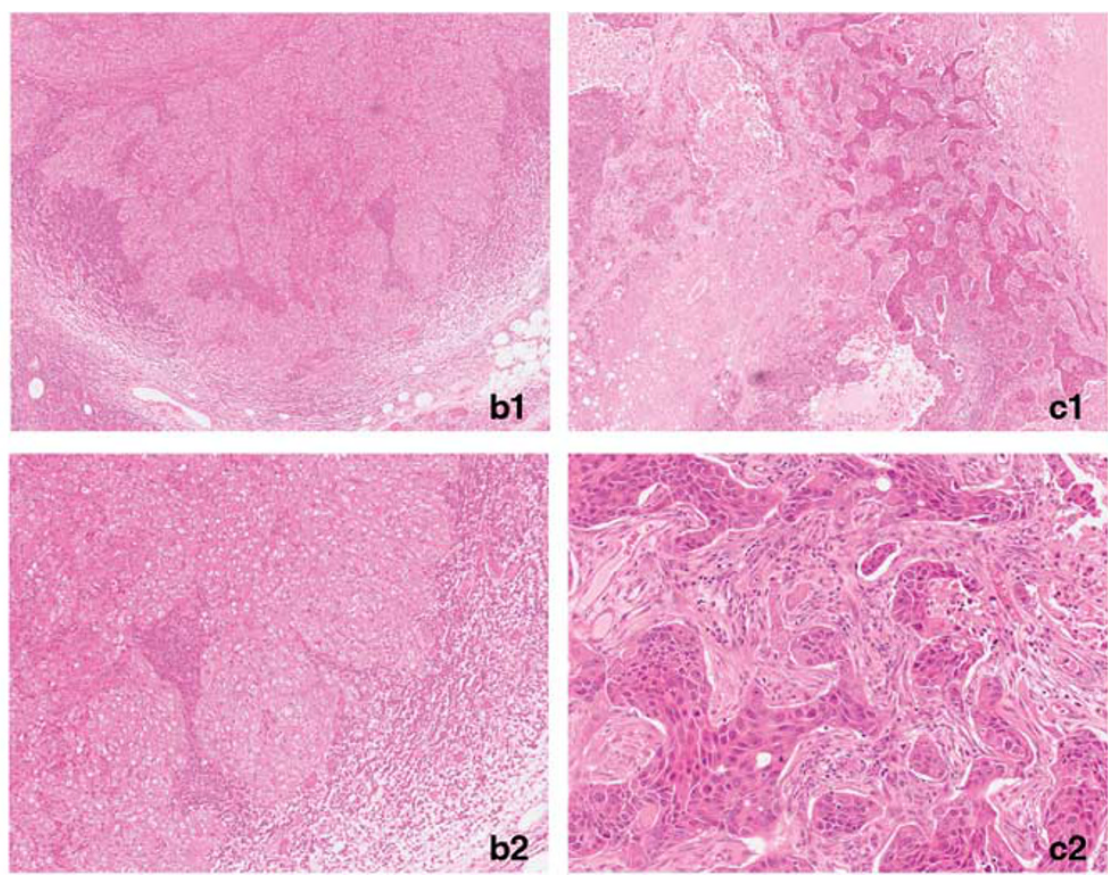

Figure 1 Histopathologic features of basal-like, medullary-like and metaplastic invasive carcinomas. (a1) Basal-like carcinoma with associated geographical necrosis (low power). (a2) High mitotic rate, apoptotic cells and necrosis (high power). (b1) Medullary-like carcinoma with pushing edges (low power). (b2) Cytologic features of medullary-like carcinoma cells with lymphoplasmacytic stromal infiltration (high power). (c1) Metaplastic tumor (low power). (c2) Spindle cells (high power). 

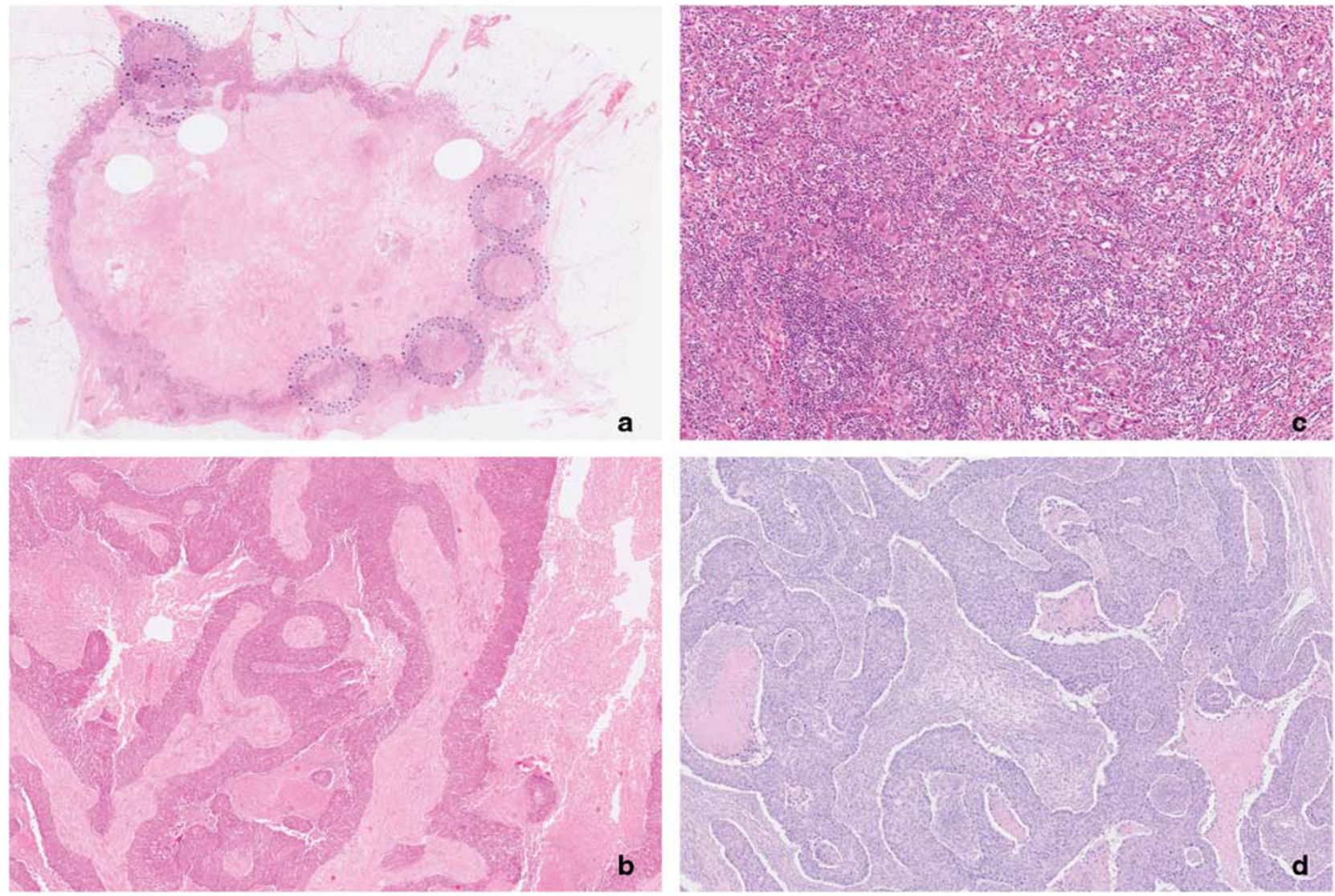

Figure 2 Appearance of tumors with distinctive fibrosis/necrosis and lymphocytic infiltrate recorded in pathological review. (a) Central fibrosis, (b) basal-like fibrosis/necrosis and (c, d) significant lymphocytic infiltration associated with invasive tumor.

Table 1 Antibody panel used for immunohistochemical profiling

\begin{tabular}{lccllc}
\hline Antigen & Clone & Dilution & Source & System & Positive score \\
\hline ER & SP1 & $1: 100$ & Invitrogen & Leica BOND-Max & $>2$ Allred \\
PR & 1A6 & $1: 300$ & Dako & Leica BOND-Max & $>2$ Allred \\
EGFR & 384 & $1: 100$ & Leica & Leica BOND-Max & $2+/ 3+$ \\
CK5/6 & D5/16 B4 & $1: 100$ & Dako & Leica BOND-Max & $>10 \%$ \\
CK14 & E3 & $1: 100$ & Dako & Leica BOND-Max & $>10 \%$ \\
HER2 & & Ready to use kit & Leica & Leica BOND-Max & $3+$ \\
HER2 & & Ready to use kit & Ventana & Ventana (CISH) & Amplification ratio $>2.0$
\end{tabular}

cran.r-project.org/). A Sweave documentation is provided in the Supplementary Methods. Risk of death was estimated on our right-censored clinical follow-up data from all 142 triple-negative breast cancers, using cumulative incidence and Cox proportional hazard models with breast cancer death as the end point; $P$-values were estimated using the Fine and Gray's test and the log-rank test, respectively. We examined the relationship between different clinicopathological variables using Fisher's exact tests. All $P$-values were two sided and a $P$-value of $<0.05$ was considered to indicate a statistically significant difference.

\section{Results}

\section{Basal Features Based on Immunohistochemical Profile}

Immunohistochemical assessment of the 142 invasive breast carcinomas (which were all confirmed as negative for the expression of ER, PR and HER2) revealed that $78(54 \%)$ were positive for EGFR, 79 (56\%) for CK5/6 and 87 (61\%) for CK14 (Figure 3); the clinicopathologic characteristics are listed in Table 3. According to the Nielsen Criteria, ${ }^{16}$ triplenegative breast cancers positive for either EGFR or CK5/6 or both were categorized as 'Core Basal' $(n=68 ; 48 \%)$, whereas $54(38 \%)$ were 5 NP. Nearly 
Table 2 Scoring system for immunohistochemistry

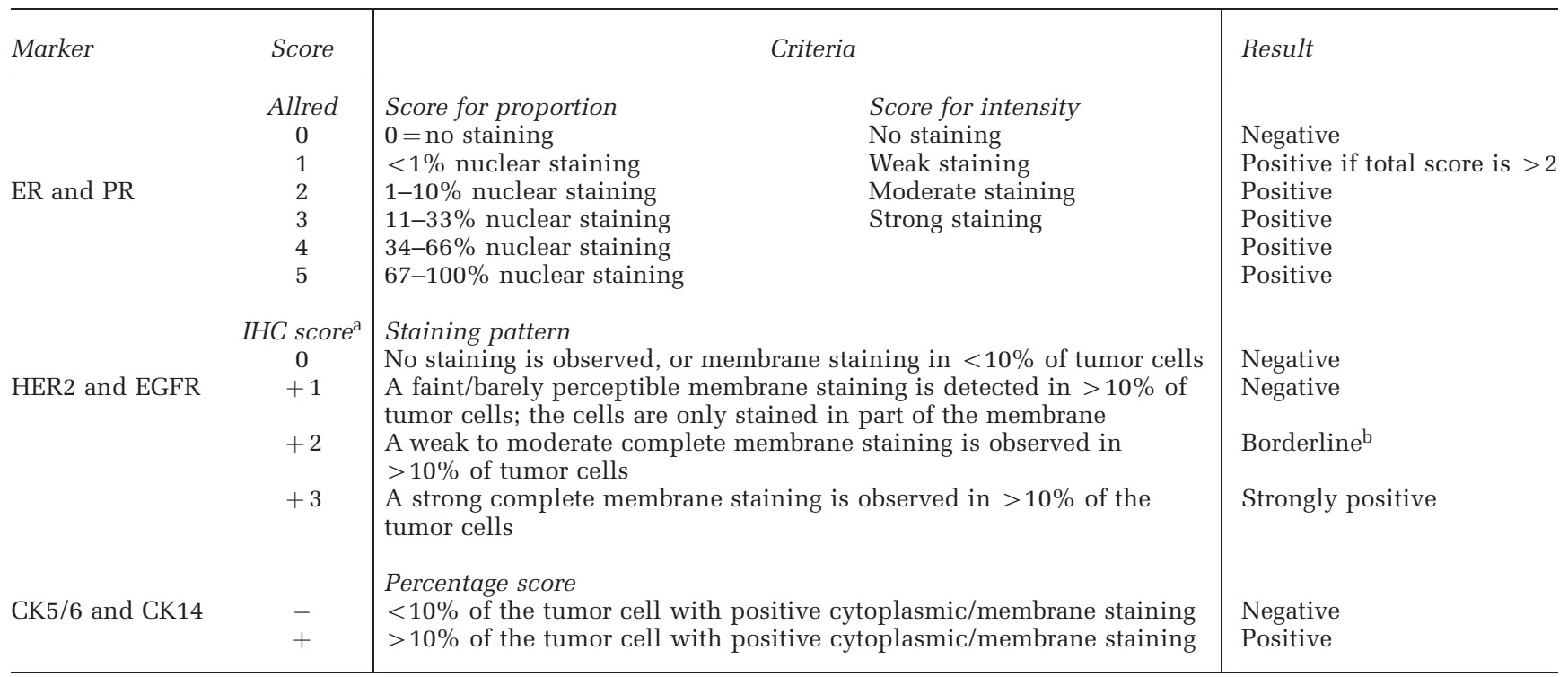

${ }^{\text {a}}$ NHSBSP January 2005-recommendation.

$\mathrm{b}+2$ result is borderline for HER2 and needs ISH validation.
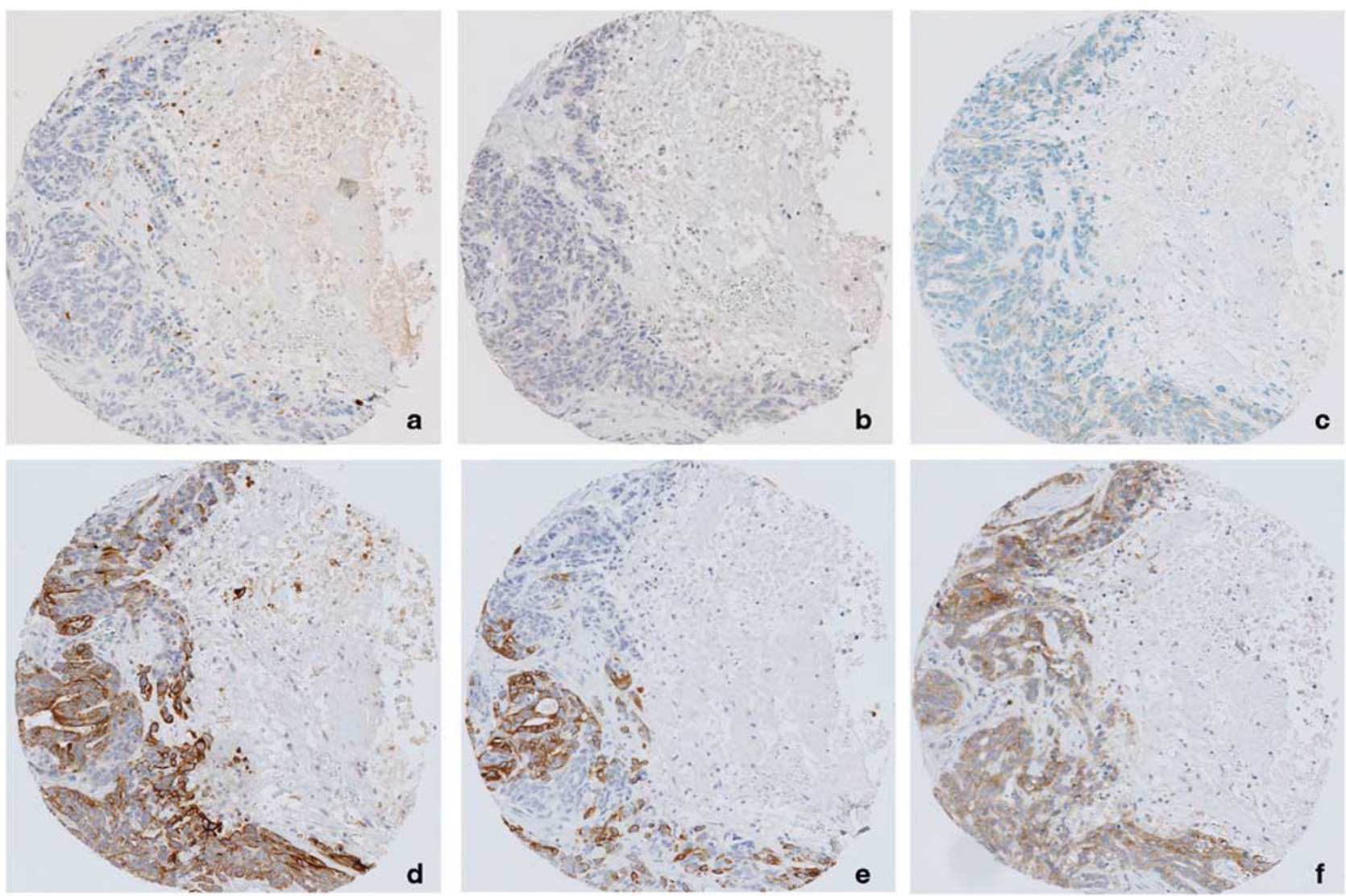

Figure 3 Immunophenotype of basal-like carcinomas: negative staining for ER, PR and HER2 and positive reaction for CK14, CK5/6 and EGFR. Immunophenotype of basal-like carcinomas: (a) ER negative, (b) PR negative, (c) HER2 negative, (d) CK14 positive, (e) CK5/6 positive and (f) EGFR positive. 
Table 3 Clinicopathological features of triple-negative breast cancer

\begin{tabular}{|c|c|c|c|c|c|c|c|c|c|c|}
\hline & $\begin{array}{c}T N B C \\
(\mathrm{n}=142)\end{array}$ & $\begin{array}{l}\text { Core Basal } \\
\quad(\mathrm{n}=68)\end{array}$ & $\begin{array}{c}5 N P \\
(\mathrm{n}=54)\end{array}$ & $\mathrm{P}$-value & $\begin{array}{l}\text { Path-Basal } \\
\quad(\mathrm{n}=34)\end{array}$ & $\begin{array}{l}\text { Path-NonBasal } \\
\quad(\mathrm{n}=101)\end{array}$ & $\mathrm{P}$-value & $\begin{array}{l}\text { PAM50-Basal } \\
\quad(\mathrm{n}=84)\end{array}$ & $\begin{array}{l}\text { PAM50-NonBasal } \\
(\mathrm{n}=17)\end{array}$ & $\mathrm{P}$-value \\
\hline \multicolumn{11}{|l|}{ Age (years) } \\
\hline$<$ Below 50 & 56 & 29 & 16 & & 12 & 42 & & 36 & 5 & \\
\hline$>50$ & 86 & 39 & 38 & 0.186 & 22 & 59 & 0.55 & 48 & 12 & 0.418 \\
\hline \multicolumn{11}{|l|}{ Menopausal status } \\
\hline Premenopausal & 60 & 30 & 17 & & 13 & 45 & & 40 & 5 & \\
\hline Postmenopausal & 72 & 36 & 30 & & 18 & 50 & & 40 & 12 & \\
\hline Perimenopausal & 8 & 2 & 5 & 0.104 & 3 & 5 & 0.668 & 4 & 0 & 0.254 \\
\hline \multicolumn{11}{|l|}{ Tumor size, $\mathrm{cm}$} \\
\hline$\leq 2$ & 29 & 13 & 10 & & 6 & 23 & & 19 & 2 & \\
\hline $2-5$ & 87 & 41 & 32 & & 24 & 58 & & 52 & 11 & \\
\hline$>5$ & 21 & 11 & 10 & 0.964 & 3 & 16 & 0.433 & 10 & 4 & 0.353 \\
\hline \multicolumn{11}{|l|}{ Lymph node status } \\
\hline Positive & 55 & 32 & 16 & & 6 & 46 & & 39 & 3 & \\
\hline Negative & 61 & 27 & 26 & & 21 & 41 & & 39 & 6 & \\
\hline Unknown & 24 & 9 & 12 & 0.156 & 7 & 14 & $<0.01$ & 6 & 8 & 0.486 \\
\hline \multicolumn{11}{|l|}{ Histological grade } \\
\hline 1 & 1 & 0 & 1 & & 0 & 1 & & 0 & 0 & \\
\hline 2 & 5 & 3 & 1 & & 1 & 3 & & 1 & 2 & \\
\hline 3 & 136 & 65 & 52 & 0.459 & 33 & 97 & 1 & 83 & 15 & 1 \\
\hline \multicolumn{11}{|l|}{ Components of grade } \\
\hline \multicolumn{11}{|l|}{ Tubule formation } \\
\hline 1 & 0 & 0 & 0 & & 0 & 0 & & 0 & 0 & \\
\hline 2 & 8 & 5 & 3 & & 0 & 8 & & 3 & 1 & \\
\hline \multirow{2}{*}{\multicolumn{11}{|c|}{ Mitotic count }} \\
\hline & & & & & & & & & & \\
\hline 1 & 4 & 2 & 2 & & 0 & 3 & & 1 & 1 & \\
\hline 2 & 8 & 4 & 3 & & 2 & 6 & & 4 & 3 & \\
\hline 3 & 98 & 43 & 37 & 1 & 29 & 64 & 0.745 & 59 & 9 & 0.058 \\
\hline \multicolumn{11}{|l|}{$\begin{array}{l}\text { Nuclear } \\
\text { pleomorphism }\end{array}$} \\
\hline 1 & 0 & 0 & 0 & & 0 & 0 & & 0 & 0 & \\
\hline 2 & 2 & 0 & 2 & & 0 & 2 & & 1 & 0 & \\
\hline 3 & 108 & 49 & 40 & 0.21 & 31 & 71 & 1 & 63 & 13 & 1 \\
\hline \multicolumn{11}{|l|}{ Histological type } \\
\hline Apocrine & 2 & 0 & 1 & & NA & NA & & 1 & 0 & \\
\hline $\begin{array}{l}\text { Morphological } \\
\text { basal-like }\end{array}$ & 28 & 15 & 7 & & NA & NA & & 19 & 2 & \\
\hline Invasive papillary & 1 & 1 & 0 & & NA & NA & & 1 & 0 & \\
\hline Lobular & 4 & 2 & 1 & & NA & NA & & 2 & 0 & \\
\hline Medullary-like & 2 & 1 & 1 & & NA & NA & & 2 & 0 & \\
\hline Metaplastic & 4 & 1 & 2 & & NA & NA & & 1 & 2 & \\
\hline Mixed & 7 & 4 & 3 & & NA & NA & & 1 & 1 & \\
\hline NST & 92 & 44 & 37 & & NA & NA & & 12 & 12 & \\
\hline Salivary gland-like & 2 & 0 & 2 & & NA & NA & & 0 & 0 & \\
\hline
\end{tabular}




\begin{tabular}{|c|c|c|c|c|c|c|c|c|c|c|}
\hline & $\begin{array}{c}\text { TNBC } \\
(\mathrm{n}=142)\end{array}$ & $\begin{array}{l}\text { Core Basal } \\
(\mathrm{n}=68)\end{array}$ & $\begin{array}{c}5 N P \\
(\mathrm{n}=54)\end{array}$ & $\mathrm{P}$-value & $\begin{array}{c}\text { Path-Basal } \\
(\mathrm{n}=34)\end{array}$ & $\begin{array}{l}\text { Path-NonBasal } \\
\quad(\mathrm{n}=101)\end{array}$ & $\mathrm{P}$-value & $\begin{array}{c}\text { PAM50-Basal } \\
(\mathrm{n}=84)\end{array}$ & $\begin{array}{l}\text { PAM50-NonBasal } \\
(\mathrm{n}=17)\end{array}$ & $\mathrm{P}$-value \\
\hline \multicolumn{11}{|l|}{$L V I$} \\
\hline Present & 35 & 14 & 18 & & 1 & 32 & & 19 & 5 & \\
\hline Absent & 107 & 54 & 36 & 0.147 & 33 & 69 & $<0.001$ & 65 & 12 & 0.543 \\
\hline \multicolumn{11}{|l|}{ Lymphoid infiltrate } \\
\hline Present & 50 & 22 & 15 & & 9 & 39 & & 34 & 3 & \\
\hline Absent & 92 & 46 & 39 & 0.692 & 25 & 62 & 0.221 & 50 & 14 & 0.993 \\
\hline \multicolumn{11}{|l|}{ Necrosis } \\
\hline Marked & 81 & 42 & 28 & & 29 & 49 & & 51 & 8 & \\
\hline Minimal or absent & 60 & 26 & 25 & 0.357 & 5 & 51 & $<0.01$ & 32 & 9 & 0.291 \\
\hline \multicolumn{11}{|l|}{ Fibrosis } \\
\hline Present & 44 & 22 & 15 & & 8 & 32 & & 23 & 7 & \\
\hline Absent & 97 & 46 & 38 & 0.693 & 26 & 68 & 0.393 & 60 & 10 & 0.383 \\
\hline \multicolumn{11}{|l|}{ DCIS } \\
\hline Present & 48 & 27 & 16 & & 8 & 38 & & 31 & 8 & \\
\hline Absent & 94 & 41 & 38 & 0.26 & 26 & 63 & 0.149 & 53 & 9 & 0.585 \\
\hline \multicolumn{11}{|l|}{ EGFR } \\
\hline Positive & 45 & 45 & 0 & & 13 & 31 & & 28 & 5 & \\
\hline Negative & 78 & 22 & 54 & & 14 & 58 & & 43 & 9 & \\
\hline Unknown & 19 & 1 & 0 & $<0.01$ & 7 & 12 & 0.259 & 13 & 3 & 1 \\
\hline \multicolumn{11}{|l|}{ CK5/6 } \\
\hline Positive & 44 & 44 & 0 & & 9 & 31 & & 31 & 1 & \\
\hline Negative & 79 & 24 & 54 & & 18 & 58 & & 40 & 13 & \\
\hline Unknown & 19 & 0 & 0 & $<0.01$ & 7 & 12 & 1 & 13 & 3 & 0.013 \\
\hline \multicolumn{11}{|l|}{ CK14 } \\
\hline Positive & 35 & 22 & 12 & & 8 & 24 & & 23 & 3 & \\
\hline Negative & 87 & 43 & 42 & & 17 & 66 & & 46 & 11 & \\
\hline Unknown & 20 & 3 & 0 & 0.221 & 9 & 11 & 0.619 & 15 & 3 & 0.532 \\
\hline \multicolumn{11}{|l|}{ Adjuvant treatment } \\
\hline Chemotherapy & 57 & 31 & 19 & 0.27 & 12 & 43 & 0.546 & 38 & 2 & 0.013 \\
\hline Endocrine & 63 & 30 & 24 & 1 & 20 & 38 & 0.044 & 31 & 11 & 0.056 \\
\hline Radiotherapy & 86 & 39 & 30 & 0.856 & 21 & 61 & 1 & 55 & 6 & 0.029 \\
\hline \multicolumn{11}{|l|}{ Metastasis site } \\
\hline Lung & 21 & 11 & 7 & 0.798 & 3 & 15 & 0.56 & 10 & 2 & 1 \\
\hline Liver & 9 & 4 & 4 & 0.731 & 0 & 8 & 0.2 & 5 & 1 & 1 \\
\hline Brain & 12 & 6 & 3 & 0.729 & 2 & 10 & 0.729 & 8 & 1 & 1 \\
\hline Bone & 19 & 8 & 10 & 0.316 & 1 & 16 & 0.07 & 8 & 4 & 0.115 \\
\hline
\end{tabular}


all of the triple-negative breast cancers (95\%) were histologic grade 3 ; thus, no difference in grade was observed between 'Core Basal' and 5NP tumors (Table 3). Of note, the only grade 1 triple-negative breast cancer was negative for EGFR, CK5 (ie, 5NP) as well as for CK14.

The majority of triple-negative breast cancers cohort ranged from 2 to $5 \mathrm{~cm}(61 \%)$ and no difference in tumor size between 'Core Basal' or $5 \mathrm{NP}$ tumors was detected. Central fibrosis, a lymphoid infiltrate and lympho-vascular invasion were observed in approximately one-third of all triple-negative breast cancers. Comparison of pathological characteristics revealed a similar distribution for most features with the exception of lymphovascular invasion, which was slightly and nonsignificantly more frequent in 'Core Basal' $(80 \%)$ than in 5NP (67\%; Fisher's exact test; $P=0.14$ ).

At the time of diagnosis, $60 \%$ of patients were $>50$ years old, and most were postmenopausal. Despite the similar age distribution of the 'Core Basal' and the 5NP group, the 5NP group included fewer premenopausal women. A slight tendency for bone metastasis was noted in patients with 5NP tumors, but no difference was observed in nodal status and treatment regime (Table 3). Overall, the immunohistochemical basal-like classification was not associated with any specific clinicopathological features in these triple-negative breast cancers.

\section{Basal Features Based on Histological Assessment}

Within this cohort of triple-negative breast cancers, a variety of different histologic subtypes were identified (Table 3); there was one case of invasive papillary and two cases each of salivary gland-like, medullary-like and apocrine-like carcinomas. Four other samples showed metaplastic and four lobular features, and seven samples had mixed morphological characteristic. Twenty-eight were morphologically basal-like and the remaining were ductal/ no-special type without morphologic basal-like features $(n=92)$. In total, 34 tumors were designated as having a basal-like morphology, incorporating the basal-like ductal/no-special types, medullary-like and metaplastic carcinomas, and referred to hereafter as 'Path-Basal'. The triple-negative breast cancers without morphological basal-like features were labeled as 'Path-NonBasal' $(n=101)$. Comparison of EGFR, CK5/6 and CK14 status in these two morphological groups indicated a similar distribution (Table 3). In contrast to the 'Core Basal' triplenegative breast cancers, lymphovascular invasion was absent in all 'Path-Basal' lesions but was observed in nearly one-third of the 'Path-NonBasal' tumors (Fisher's exact test; $P<0.001$ ). Moreover, $17 \%$ of 'Path-Basal' cancers had a positive nodal status compared with $45 \%$ of 'Path-NonBasal' lesions (Fisher's exact test; $P<0.001$ ). The most significant difference was the presence of geographic necrosis, which was seen in $85 \%$ 'PathBasal', but only in $48 \%$ 'Path-NonBasal' lesions (Fisher's exact test; $P<0.001$ ). As necrosis represents one of the diagnostic features to define the basal-like ductal/no-special type lesions, this was to be anticipated.

\section{Molecular 'Basal-Like’ Classification}

In addition to the immunohistochemical and histological assessments of basal-like lesions, we were able to assign the molecular basal-like subtype based on the PAM50 centroid classification to 84 triplenegative breast cancers (see Materials and Methods). These tumors are referred to as 'PAM50-Basal' hereafter.

\section{Overlap of Basal-Like Breast Carcinomas Defined by Different Criteria}

Comparing the tumors classified as basal-like by these three different methodologies ('Core Basal', 'Path-Basal' and 'PAM50-Basal') identified 116/142 triple-negative breast cancer as basal-like by at least one assessment. The overlap of tumors categorized as basal-like is shown in Figure 4a. Only 13 cases were defined as basal-like by all three methods (Figure 4). Of these, 9 samples were EGFR positive, 7 were CK5/6 positive, 5 were CK14 positive, 5 had a positive nodal status, 4 had associated DCIS and fibrosis and 2 showed lymphocytic infiltration. It is noteworthy that all 13 samples lacked associated lymphovascular invasion, whereas 35 of the remaining 109 lesions (32\%) had lymphovascular invasion identified (Fisher's exact test; $P=0.038$ ).

Given that the immunohistochemical panel proposed by Nielsen et $a l^{7}$ is considered to be the gold standard by some authors, we established how many of the 'Core Basal' lesions would have been categorized as basal-like by morphological or molecular assessments. Only 17/68 'Core Basal' triple-negative breast cancers were assigned as 'Path-Basal', suggesting a low sensitivity (26\%) for the histologic assessment to identify 'Core Basal' lesions (Table 4a). In contrast, the 'PAM50-Basal' classification assigned 44/68 'Core Basal' triplenegative breast cancers to the basal-like molecular subtype (Table 4b), showing $64 \%$ sensitivity but a low specificity of $51 \%$ to identify nonbasal lesions.

\section{Risk of Breast Cancer-Specific Death}

To assess if the breast carcinomas defined as basallike by these three diverse methods differed in predicting prognosis, the cumulative incidence of breast cancer death was estimated. Triple-negative breast cancers categorized as 'Core Basal' (Figure 4b) showed an increased risk of death within 15 years after diagnosis compared with those assigned 
a

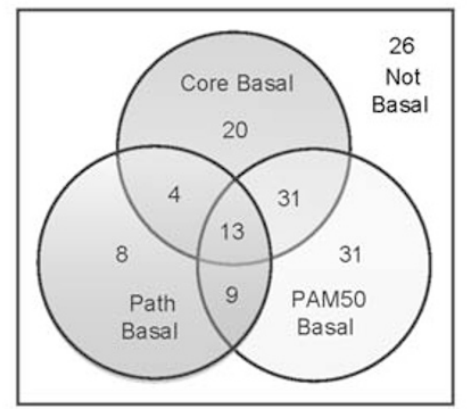

C

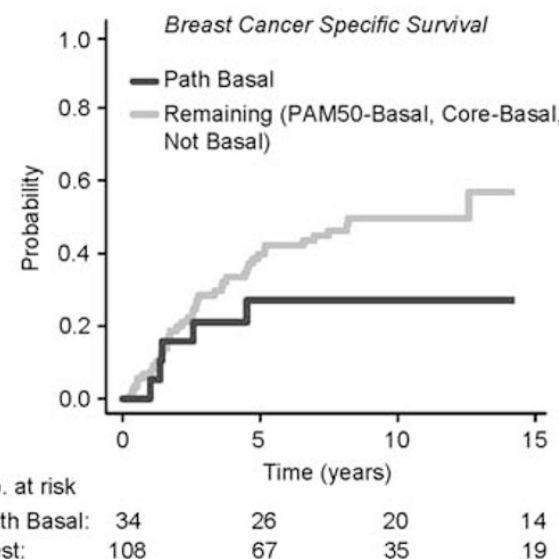

b

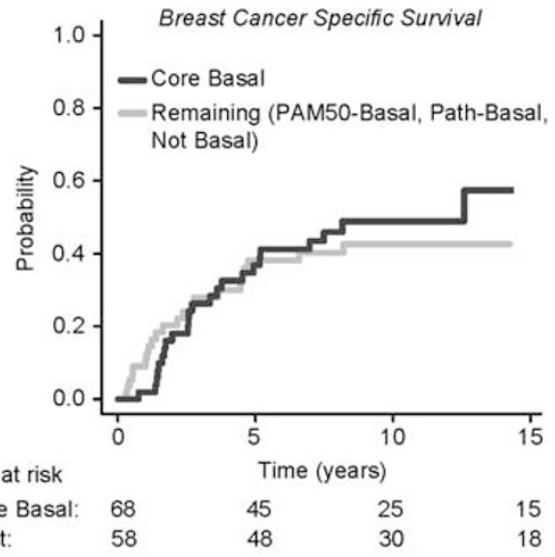

d

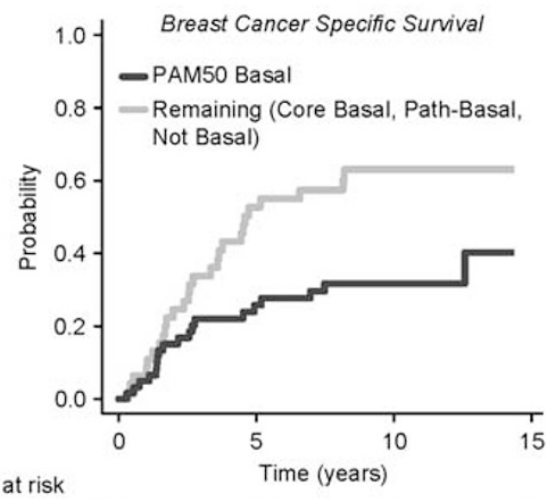

No. at risk

PAM50 Basal: $84 \quad 62 \quad 38 \quad 22$

Figure 4 Comparison of triple-negative breast cancers defined as 'basal-like' by histopathology, immunoprofiling and genomics. (a) A Venn diagram, showing the overlap of triple-negative breast cancer cases assigned by the three different methods. Lesions not assigned to the basal-like phenotype by any of the three methods are referred to as 'Not Basal'. The 15-year cumulative incidence curves of breast cancer-specific mortalities since the time of diagnosis are displayed for 'Core Basal' (b), 'PAM50-Basal (c) and 'Path-Basal' (d). For each graph, the 'basal-like' features were compared with a reference group, called 'Remaining', which consisted of all other triplenegative breast cancers except the respective 'basal-like' category in question. The compilation of the 'Remaining' is given in brackets in each analysis. The number of patients at risk for 5, 10 and 15 years is given for each subgroup.

Table 4 Comparison of morphologic and molecular basal-like assessment with immunohistochemical groups

\begin{tabular}{lcc}
\hline & Core Basal & $5 N P$ \\
\hline (a) IHC and morphological Assessment & \\
Path-Basal & 17 & 10 \\
Path-NonBasal & 47 & 41 \\
& Sensitivity: $26 \%$ & Specificity: $80 \%$ \\
(b) IHC and molecular Assessment & \\
PAM50-Basal & 44 & 26 \\
PAM50-NonBasal & 24 & 28 \\
& Sensitivity: $64 \%$ & Specificity: $51 \%$ \\
\hline
\end{tabular}

as 'Path-Basal' (Figure 4c) or 'PAM50-Basal' (Figure 4d). However, those characterized as 'Core Basal' did not have a statistically significantly different outcome than those not 'Core Basal' (Figure 4b), whereas the two other characterizations separated patients with regard to prognosis (Figure 4c and d); this finding was also supported by the results of the Cox Regression analysis
(Table 5). A significantly better 15-year breast cancer-specific survival was seen for women with tumors in the 'PAM50-Basal' and the 'Path-Basal' groups compared with the remaining samples; this maintained significant independent prognostic value to predict breast cancer death in the different multivariate Cox models (Table 5).

\section{Discussion}

The terminology, definition and classification of basal-like breast cancer are controversial and vary in the literature, making comparison of clinicopathologic features difficult. Different terms are used to describe these tumors as 'basal-like', 'basal', 'Core Basal' and even 'basaloid', as well as those with a 'basal/myoepithelial phenotype', and it is unclear if the same lesions are identified. In order to assess the degree of overlap in identifying basal-like features in triple-negative breast cancer, we examined a set of triple-negative breast cancers in a series of women who received no neoadjuvant therapy. We defined a 
Table 5 Cox regression analysis to estimate the adjusted hazard ratios (HRs) of 142 triple-negative breast cancers with sufficient information for all of the variables, classified as basal-like by three different methods

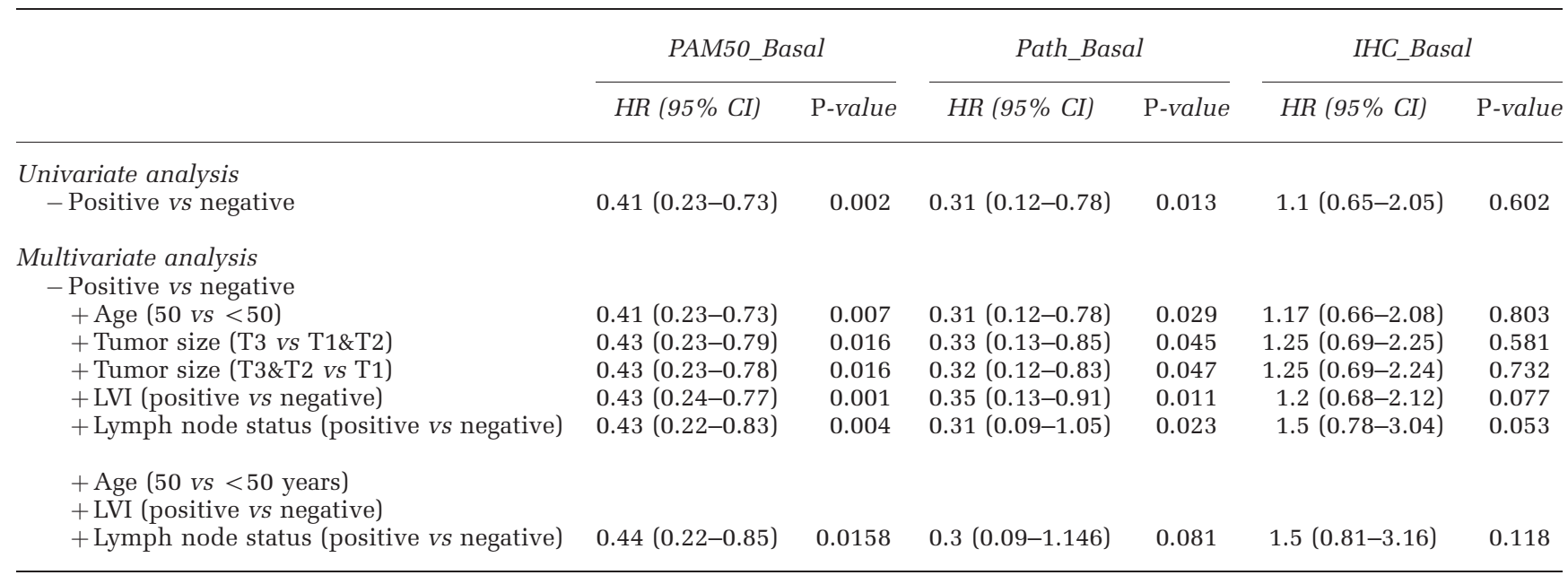

HRs with their 95\% confidence intervals (CIs) for being positive versus negative in the respective classification in each model are shown.

morphological group of triple-negative breast cancers with histologic basal-like features ('Path-Basal') and compared these with the immunohistochemical ('Core Basal') and gene expression profiles ('PAM50Basal') of the same lesions with regard to their clinicopathologic features and patient outcome.

The majority of morphological classified 'PathBasal' triple-negative breast cancers in this study showed geographic necrosis, in comparison with only $48 \%$ 'Path-NonBasal' lesions. Extensive central necrosis has been reported as more marked in tumors lacking hormone receptor and those occurring in younger patients. ${ }^{9}$ It is also reported in a subset of lesions showing poor response to chemotherapy and endocrine therapy, possibly as a result of poor penetration of therapeutic agents into the tumor mass. ${ }^{26}$ Despite the presence of necrosis, which is commonly associated with poor prognosis, triple-negative breast cancers classified as 'PathBasal' had a better outcome (Figure 4c). Although this must be interpreted with caution, as patient numbers are small, the lack of lymph node metastases in the 'Path-Basal' group might also contribute to this tendency. In addition, the 'Path-Basal' samples, as well as the 13 lesions defined as basallike by all three methods, were characterized by an absence of lymphovascular invasion. Lymphovascular invasion has been reported to be an independent prognostic factor for poor outcome in triplenegative breast cancers and lymph node-negative and ER-negative breast cancers, ${ }^{27}$ supporting the lack of lymphovascular invasion in the 'Path-Basal' group having a lower risk of breast cancer-specific death. Interestingly, the same trend for a better prognosis for basal-like lesions was observed in the 'PAM50-Basal' group when compared with the remainder of the triple-negative breast cancers in this series and this remained prognostically significant when other potential confounding clinico- pathologic variables were taken into consideration. This is in contrast to some previous studies, showing that the expression of immunohistochemical basal markers correlates with a worse prognosis. ${ }^{16}$ However, recently, 'Core Basal' lesions among triple-negative breast cancers with high CD8 + infiltrating lymphocytes ${ }^{28}$ and a Korean cohort $^{29}$ have also been reported to be associated with a better prognosis. These differences highlight the heterogeneity within triple-negative breast cancers, and the potential for identifying basal-like lesions with a good and a bad prognosis. Only further characterization such as the prevalence of CD8 + lymphocytes in our cohort might shed further light on this matter and is warranted.

Comparison between gene expression and immunohistochemical methods to define basal-like tumors have been performed previously, and $76 \%$ sensitivity and $100 \%$ specificity of immunohistochemical surrogates to identify the molecular basallike subtype has been reported. ${ }^{7}$ Using an extended panel of immunohistochemical markers including CK14, EGFR and $34 \beta$ E12, a slightly increased sensitivity $(78 \%)$ for immunohistochemistry to detect molecularly defined basal-like tumors has been identified. ${ }^{30}$ Although our specificity measurements are lower than these previous observations, we have seen a similar proportion of nonbasal invasive breast cancers by the morphologic and immunohistochemical methods in those tumors identified as basal-like by molecular assessments $(60 \%)$. In contrast, we find a weak specificity (26\%) for the 'Path-Basal' and a slightly better one (59\%) using 'Core Basal' to capture 'PAM50-Basal', suggesting poor agreement between these descriptive methods.

The recognition of basal-like tumors is potentially clinically important. Some series have suggested that, in lymph node-negative breast cancer in 
particular, the basal phenotype is of independent prognostic value, whereas ER/PR expression is not prognostically significant. ${ }^{17}$ Clearly, accurate determination of ER and HER2 status is necessary to identify clinical targets and direct therapy. The immunohistochemical assessment of basal cytokeratins and EGFR is not routine and does not form part of invasive breast cancer pathology minimum data sets. Nevertheless, our data indicate that morphologic assessment is insufficient to identify those patients with triple-negative breast cancers with the worst outcome (and only identified 34 basal-like tumors) whereas patients with tumors defined as 'Core Basal' had the poorest breast cancer-specific survival, further strengthening the inclusion of EGFR and basal cytokeratins as biomarkers to predict prognosis. ${ }^{16}$

Study limitations include the fact that this cohort of triple-negative breast cancers is relatively small, derived from consecutive cases from one hospital over a period of many years and that systemic treatments were not randomized, making outcomes stratified by treatment difficult to interpret. In particular, as treatment regimens were heterogeneous, we acknowledge a diverse influence on the outcome data. In addition, it is possible that some discrepancies could be attributed to the use of tissue microarrays for immunohistochemical evaluation, as intratumoral heterogeneity for basal cytokeratin expression has been observed. ${ }^{31}$

However, this study does illustrate that the identification and frequency of classification of basal-like invasive breast cancer depends on the methodology used. Importantly, we show that the lesions identified differ in their clinicopathologic features. Although many studies and clinical trials use molecular classification to define basal-like breast cancer, it is possible that this approach lacks specificity and overestimates the prevalence of the basal-like phenotype in triple-negative breast cancer. Our findings stress the need for clarity in publications regarding methodology and corroborate previous observations that triple-negative breast cancers and basal-like breast cancer are not synonymous. This study highlights the need to define a routinely assessable series of characteristics (potentially an admixture of morphologic and immunohistochemical biomarkers) characterizing this category of invasive carcinomas.

\section{Acknowledgements}

Anita Grigoriadis and Patrycja Gazinska are supported by the Breakthrough Breast Research Unit funding at King's College London. This research was supported by the Experimental Cancer Medicine Centre at King's College London, the National Institute for Health Research Biomedical Research Centre based at Guy's and St Thomas' NHS Foundation Trust and King's College London and
Breakthrough Breast Research. Patient tissue samples were provided by Guy's and St Thomas' Breast Tissue and Data Bank, which is supported by the Department of Health via the National Institute for Health Research comprehensive Biomedical Research Centre award. Gene expression data on the tissue samples were provided by Breakthrough Breast Research Unit.

\section{Disclosure/conflict of interest}

The authors declare no conflict of interest.

\section{References}

1 Foulkes WD, Smith IE, Reis-Filho JS. Triple-negative breast cancer. N Engl J Med 2010;363:1938-1948.

2 Rakha E, Reis-Filho JS. Basal-like breast carcinoma: from expression profiling to routine practice. Arch Pathol Lab Med 2009;133:860-868.

3 Subhawong AP, Subhawong T, Nassar H, et al. Most basal-like breast carcinomas demonstrate the same $\mathrm{Rb}-/ \mathrm{p} 16+$ immunophenotype as the HPV-related poorly differentiated squamous cell carcinomas which they resemble morphologically. Am J Surg Pathol 2009;33:163-175.

4 Carey LA, Dees EC, Sawyer L, et al. The triple negative paradox: primary tumor chemosensitivity of breast cancer subtypes. Clin Cancer Res 2007;13:2329-2334.

5 Morris GJ, Naidu S, Topham AK, et al. Differences in breast carcinoma characteristics in newly diagnosed African-American and Caucasian patients: a singleinstitution compilation compared with the National Cancer Institute's Surveillance, Epidemiology, and End Results database. Cancer 2007;110:876-884.

6 Brenton JD, Carey LA, Ahmed AA, et al. Molecular classification and molecular forecasting of breast cancer: ready for clinical application? J Clin Oncol 2005;23:7350-7360.

7 Nielsen TO, Hsu FD, Jensen K, et al. Immunohistochemical and clinical characterization of the basal-like subtype of invasive breast carcinoma. Clin Cancer Res 2004;10:5367-5374.

8 Sorlie T, Perou CM, Tibshirani R, et al. Gene expression patterns of breast carcinomas distinguish tumor subclasses with clinical implications. Proc Natl Acad Sci USA 2001;98:10869-10874.

9 Fulford LG, Reis-Filho JS, Ryder K, et al. Basal-like grade III invasive ductal carcinoma of the breast: patterns of metastasis and long-term survival. Breast Cancer Res 2007;9:R4.

10 Dent R, Trudeau M, Pritchard KI, et al. Triple-negative breast cancer: clinical features and patterns of recurrence. Clin Cancer Res 2007;13:4429-4434.

11 Perou CM, Sorlie T, Eisen MB, et al. Molecular portraits of human breast tumours. Nature 2000;406: $747-752$.

12 Bertucci F, Finetti P, Cervera N, et al. How basal are triple-negative breast cancers? Int J Cancer 2008;123: 236-240.

13 Badve S, Dabbs DJ, Schnitt SJ, et al. Basal-like and triple-negative breast cancers: a critical review with an emphasis on the implications for pathologists and oncologists. Mod Pathol 2011;24:157-167. 
14 Rakha EA, Reis-Filho JS, Ellis IO. Basal-like breast cancer: a critical review. J Clin Oncol 2008;26: 2568-2581.

15 Reis-Filho JS, Tutt AN. Triple negative tumours: a critical review. Histopathology 2008;52:108-118.

16 Cheang MC, Voduc D, Bajdik C, et al. Basal-like breast cancer defined by five biomarkers has superior prognostic value than triple-negative phenotype. Clin Cancer Res 2008;14:1368-1376.

17 Rakha EA, El-Sayed ME, Green AR, et al. Prognostic markers in triple-negative breast cancer. Cancer 2007; 109:25-32.

18 Livasy CA, Karaca G, Nanda R, et al. Phenotypic evaluation of the basal-like subtype of invasive breast carcinoma. Mod Pathol 2006;19:264-271.

19 Haupt B, Ro JY, Schwartz MR. Basal-like breast carcinoma: a phenotypically distinct entity. Arch Pathol Lab Med 2010;134:130-133.

20 Da Silva L, Clarke C, Lakhani SR. Demystifying basallike breast carcinomas. J Clin Pathol 2007;60:1328-1332.

21 Putti TC, El-Rehim DM, Rakha EA, et al. Estrogen receptor-negative breast carcinomas: a review of morphology and immunophenotypical analysis. Mod Pathol 2005;18:26-35.

22 Winter J. Morphological and immunophenotypic analysis of basal-like carcinoma of the breast. Biosci Horiz 2008;1:19-27.

23 Ellis IO, Pinder SE, Bobrow L, et al. Pathology reporting of breast disease. NHSNBS 2005;58:1-144.

24 Harvey JM, Clark GM, Osborne CK, et al. Estrogen receptor status by immunohistochemistry is superior to the ligand-binding assay for predicting response to adjuvant endocrine therapy in breast cancer. J Clin Oncol 1999;17:1474-1481.

25 Parker JS, Mullins M, Cheang MC, et al. Supervised risk predictor of breast cancer based on intrinsic subtypes. J Clin Oncol 2009;27:1160-1167.

26 Maiorano E, Regan MM, Viale G, et al. Prognostic and predictive impact of central necrosis and fibrosis in early breast cancer: results from two International Breast Cancer Study Group randomized trials of chemoendocrine adjuvant therapy. Breast Cancer Res Treat 2010;121:211-218.

27 Rakha EA, Martin S, Lee AH, et al. The prognostic significance of lymphovascular invasion in invasive breast carcinoma. Cancer 2012;118: 3670-3680.

28 Liu S, Lachapelle J, Leung S, et al. CD8 + lymphocyte infiltration is an independent favorable prognostic indicator in basal-like breast cancer. Breast Cancer Res 2012;14:R48.

29 Choi YL, Oh E, Park S, et al. Triple-negative, basal-like, and quintuple-negative breast cancers: better prediction model for survival. BMC Cancer 2010;10:507.

30 Thike AA, Cheok PY, Jara-Lazaro AR, et al. Triplenegative breast cancer: clinicopathological characteristics and relationship with basal-like breast cancer. Mod Pathol 2010;23:123-133.

31 Jumppanen M, Gruvberger-Saal S, Kauraniemi P, et al. Basal-like phenotype is not associated with patient survival in estrogen-receptor-negative breast cancers. Breast Cancer Res 2007;9:R16.

Supplementary Information accompanies the paper on Modern Pathology website (http://www.nature.com/ modpathol) 